$$
a^{2}=\left(\begin{array}{rrr}
0 & 1 & 0 \\
0 & 0 & 1 \\
2 & 1 & -1
\end{array}\right), \quad a^{3}=\left(\begin{array}{rrr}
0 & 0 & 1 \\
2 & 1 & -1 \\
-2 & 1 & 2
\end{array}\right)
$$

is an example of matrices satisfying the hypotheses.

\title{
REFERENCES
}

1. E. Cartan, Sur la structure de groupes infinis de transformations, Annales Scientifiques de l'Ecole Normale Supérieure vol. 21 (1904) pp. 153-206.

2. H. H. Johnson, On terminating prolongation procedures, Pacific J. Math. vol. 10 (1960) pp. 577-583.

3. M. Kuranishi, On E. Cartan's prolongation theorem of exterior differential systems, Amer. J. Math. vol. 79 (1957) pp. 1-47.

4. C. F. Moppert, On the notion of analyticity, Proc. Amer. Math. Soc. vol. 10 (1959) pp. 574-576.

Princeton University

\section{THE POLYNOMIAL DERIVATIVE AT A ZERO ANGLE ${ }^{1}$}

W. E. SEWELL

1. Introduction. The purpose of this note is to prove the following theorem:

Let $C$ be a piecewise analytic Jordan curve bounding the simply connected region $R$ and let $z_{0}$ be a corner of $C$ with exterior angle zero. Let the 1 st, $2 n d, \cdots$, th thential derivatives of the two arcs of $C$ at $z_{0}$ vanish. Let $P_{n}(z)$ be a polynomial of the degree $n$ in $z$ such that $\left|P_{n}(z)\right|$ $\leqq M, z$ on $C$; then $\left|P_{n}^{\prime}\left(z_{0}\right)\right| \leqq M K(\log n)^{1 / t}$, where $K$ is a constant depending only on $C$.

This problem has an interesting history. In 1889 A. Markoff ${ }^{2}$ proved that if $\left|P_{n}(z)\right| \leqq M,-1 \leqq z \leqq+1$, then $\left|P_{n}^{\prime}(z)\right| \leqq M n^{2}$. And since that time notable contributions are due to $M$. Riesz, S. Bernstein, G. Szegö, J. L. Walsh, and others. For example, if the exterior angle at $z_{0}$ on the curve $C$ of the theorem is $\alpha \pi$, G. Szegö $\ddot{o}^{3}$ has shown that for $0<\alpha \leqq 2$ we have $\left|P_{n}^{\prime}\left(z_{0}\right)\right| \leqq M K n^{\alpha}$. He has also shown that

Received by the editors March 29, 1960 and, in revised form, May 11, 1960.

1 This work is sponsored by the Office of Ordnance Research, U. S. Army.

2 On a problem of $D$. I. Mendeljeff (in Russian), Bulletin of the St. Petersburg Academy vol. 62 (1890) pp. 1-24.

- Über einen Satz von A. Markoff, Math. Z. vol. 23 (1925) pp. 45-61. 
if $\left|P_{n}(z)\right| \leqq 1$ in two unit circles tangent externally at $z_{0}$ then $\left|P_{n}^{\prime}\left(z_{0}\right)\right| \leqq M K \log n$.

The author ${ }^{4}$ has studied the problem for various types of curves, viz., the ellipse, cardioid, rose curve, lemniscate, etc.; however the method used there did not yield a sharp result for the corner itself. The method presently employed consists of constructing a curve, which we call a clover leaf, whose center is at $z_{0}$ and which lies on or interior to $C$, and of mapping the exterior of the clover leaf on the exterior of the unit circle. The mapping function is constructed from the function used by Szegö in proving his two-circle theorem.

In the final paragraph we consider the case where $C$ is a classical cardioid and show that at the corner $z_{0}$ we have $\left|P_{n}^{\prime}\left(z_{0}\right)\right|$ $\leqq M K(\log n)^{2}$.

2. The four-leaf clover. In the complex plane let $z=r e^{i \theta}$ and let the curve $C$ be represented by the following equations in polar coordinates:

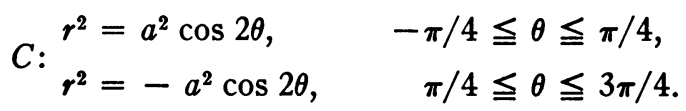$$
a^{2}>0,
$$

We shall call this curve a 4-leaf clover. It is actually two classical polar coordinate lemniscates with the same centers, and with axes perpendicular; and it is similar in shape to the 4-leaf rose. It should be noted that it is not the locus:

$$
|(z-1)(z+1)(z-i)(z+i)|=1,
$$

which is commonly called a lemniscate in complex function theory.

In the equations (1) of $C$ let $r^{2}=\rho, 2 \theta=\psi$; then curve $C$ in the $(r, \theta)$-plane becomes the curve

$$
\Omega: \begin{array}{lr}
\rho=a^{2} \cos \psi, & -\pi / 2 \leqq \psi \leqq \pi / 2, \\
\rho=-a^{2} \cos \psi, & \pi / 2 \leqq \psi \leqq 3 \pi / 2,
\end{array}
$$

which consists of two circles with centers at $\left(a^{2} / 2,0\right)$ and $\left(-a^{2} / 2,0\right)$, and radii $a^{2} / 2$. If we put $u=\rho e^{i \psi}$, the transformation is expressed by the equation

$$
z^{2}=u \text {. }
$$

For simplicity let $a^{2}=2$; then $\Omega$ consists of the two circles $|u-1|=1$ and $|u+1|=1$, which have unit radii and are tangent externally at the origin.

4 See, e.g., Degree of approximation by polynomials in the complex domain, Annals of Mathematics Studies, no. 9, Princeton, 1942, pp. 33-40, 75-78. 
The function

$$
w=i \frac{1+\exp (-i \pi / u)}{1-\exp (-i \pi / u)}
$$

maps the exterior of $\Omega$ on $|w|>1$, so that the points at $\infty$ correspond to each other. Hence the function

$$
w=g(z)=i \frac{1+\exp \left(-i \pi / z^{2}\right)}{1-\exp \left(-i \pi / z^{2}\right)}
$$

maps the exterior of the 4-leaf clover curve $C$ on $|w|>1$, so that the points at $\infty$ correspond to each other. Furthermore the quadruple point 0 in the $z$-plane corresponds to the points $w=i$ and $w=-i$, each counted twice.

We know from Cauchy's formula that

$$
P_{n}^{\prime}(0)=\frac{1}{2 \pi i} \int_{G} \frac{P_{n}(t) d t}{t^{2}},
$$

where $G$ is a circle of radius $\gamma$ with center at the origin. Hence

$$
\left|P_{n}^{\prime}(0)\right| \leqq \frac{1}{\gamma} \max _{|z|=\gamma}\left|P_{n}(z)\right| \text {. }
$$

Since the function $P_{n}(z) /[g(z)]^{n}$ is analytic exterior to the clover leaf curve $C$ and continuous on $C$, with the branches appropriately defined, then

$$
\begin{aligned}
\left|P_{n}(z)\right| \leqq M|[g(z)]|^{n} & =M|1+(w-i) / i|^{n} \\
& =M|1-(w+i) / i|^{n}
\end{aligned}
$$

for $z$ on or exterior to $C$.

Now let $0<\gamma<1$ and consider the points $z$ for which $|z|=\gamma$ and which lie outside of $C$ in the first quadrant $\left(z^{2}\right.$ is then in the upper half-plane). Then ${ }^{5}$

$$
\left|\exp \left(-i \pi / z^{2}\right)\right|=\exp \left(\left[-\pi / \gamma^{4}\right] \operatorname{Im} z^{2}\right),
$$

and the minimum ${ }^{6}$ of

$$
\operatorname{Im} z^{2}=\gamma^{2}\left(1-\gamma^{4} / 4\right)^{1 / 2} .
$$

Thus

$\checkmark$ See Szegö, loc. cit.

- If we put $z^{2}=x+i y=\gamma^{2} e^{i \phi}$, with $z$ on $C$, then $y=\gamma^{2} \sin \phi=\gamma^{2}\left(1-\cos ^{2} \phi\right)^{1 / 2}$ $=\gamma^{2}\left(1-\gamma^{4} / 4\right)^{1 / 2}$, since $\cos \phi=\gamma^{2} / 2$ for $z^{2}$ on a circle of unit radius passing through the origin and with center on the $x$-axis. 


$$
\begin{aligned}
|w-i| & =2\left|\frac{\exp \left(-i \pi / z^{2}\right)}{1-\exp \left(-i \pi / z^{2}\right)}\right| \leqq \frac{2 \exp \left(\left[-\pi / \gamma^{2}\right]\left[1-\gamma^{4} / 4\right]^{1 / 2}\right)}{1-\exp \left(\left[-\pi / \gamma^{2}\right]\left[1-\gamma^{4} / 4\right]^{1 / 2}\right)} \\
& \leqq K_{1} \exp \left(\left[-\pi / \gamma^{2}\right]\left[1-\gamma^{4} / 4\right]^{1 / 2}\right) .
\end{aligned}
$$

The same evaluation holds for $z$ in the third quadrant because $z^{2}$ is likewise in the upper half-plane.

For $z$ in the second or fourth quadrant ( $z^{2}$ in the lower half-plane), we have, as before

$$
\left|\exp \left(-i \pi / z^{2}\right)\right|=\exp \left(\left[-\pi / \gamma^{4}\right] \operatorname{Im} z^{2}\right),
$$

but here the maximum of

$$
\operatorname{Im} z^{2}=-\gamma^{2}\left(1-\gamma^{4} / 4\right)^{1 / 2} .
$$

And we consider $w-(-i)=w+i$,

$$
\begin{aligned}
|w+i| & =\left|\frac{2 i}{1-\exp \left(-i \pi / z^{2}\right)}\right| \leqq \frac{2}{\left|\exp \left(-i \pi / z^{2}\right)-1\right|} \\
& =\frac{2}{\exp \left(\left[-\pi / \gamma^{4}\right] d z^{2}\right)-1} .
\end{aligned}
$$

And since $d z^{2} \leqq-\gamma^{2}\left(1-\gamma^{4} / 4\right)^{1 / 2}$, we have

$$
\begin{aligned}
\frac{2}{\exp \left(\left[-\pi / \gamma^{4}\right] d z^{2}\right)-1} & \leqq \frac{2}{\exp \left(\left[\pi / \gamma^{2}\right]\left[1-\gamma^{4} / 4\right]^{1 / 2}\right)-1} \\
& =\frac{2 \exp \left(\left[-\pi / \gamma^{2}\right]\left[1-\gamma^{4} / 4\right]^{1 / 2}\right)}{1-\exp \left(\left[-\pi / \gamma^{2}\right]\left[1-\gamma^{4} / 4\right]^{1 / 2}\right)}
\end{aligned}
$$

or

$$
|w+i| \leqq K_{2} \exp \left(\left[-\pi / \gamma^{2}\right]\left[1-\gamma^{4} / 4\right]^{1 / 2}\right)
$$

as above.

Now if we put $\gamma=(\pi / \log n)^{1 / 2}$, we have, for $n$ sufficiently large,

$$
\left|P_{n}^{\prime}(0)\right| \leqq M(\log n)^{1 / 2}\left[1+K_{3} \exp (-\log n)\right]^{n} \leqq M K[\log n]^{1 / 2} \text {. }
$$

3. The $2 t$-leaf clover. If the curve $C$ is represented by the equations

$$
r^{t}=a^{2} \cos t \theta, \quad-\pi / 2 t \leqq \theta \leqq \pi / 2 t, 3 \pi / 2 t \leqq \theta \leqq 5 \pi / 2 t, \cdots,
$$

C:

$$
(4 t-5) \pi / 2 t \leqq \theta \leqq(4 t-3) \pi / 2 t ;
$$

$$
\begin{aligned}
r^{t}=-a^{2} \cos t \theta, \quad \pi / 2 t & \leqq \theta \leqq 3 \pi / 2 t, \cdots,(4 t-3) \pi / 2 t \leqq \theta \\
& \leqq(4 t-1) \pi / 2 t,
\end{aligned}
$$


the transformation (3) of paragraph 2 becomes

$$
z^{t}=u \text {. }
$$

And we have by substituting $t$ for 2 in the evaluation above,

$$
\left|P_{n}^{\prime}(0)\right| \leqq M K(\log n)^{1 / t} .
$$

4. The piecewise analytic curve. Now consider the curve $C$ of the theorem. Without loss of generality we can let $z_{0}$ be the origin, and the external tangent the positive $y$-axis. Then we can construct a clover-leaf curve whose equations are

$$
\Gamma: \begin{aligned}
& r^{t}=a^{2} \cos t \theta, \\
& r^{t}=-a^{2} \cos t \theta,
\end{aligned}
$$

which has its center at the origin and, for sufficiently small $a^{2}$, lies on or interior to $C$. Then $\left|P_{n}(z)\right| \leqq M, z$ on $\Gamma$, and hence $\left|P_{n}^{\prime}(0)\right|$ $\leqq M K(\log n)^{1 / t}$.

5. The cardioid. In the opposite direction the case of the cardioid is worthy of special notice. Using the notation of paragraph 3, we have

$$
\begin{aligned}
& r=\left(a^{2} / 2\right)(1-\cos \theta), \text { or } \\
& r=a^{2}\left[((1 / 2)(1-\cos \theta))^{1 / 2}\right]^{2}=a^{2} \sin ^{2}(\theta / 2) .
\end{aligned}
$$

And if we put $r=\rho^{2}, \theta=2 \psi$, equations (4) become

$$
\rho^{2}=a^{2} \sin ^{2} \psi,
$$

or

$$
\begin{aligned}
& \rho=a \sin \psi, \\
& \rho=-a \sin \psi,
\end{aligned}
$$

or two tangential circles.

Hence the transformation becomes

$$
z=u^{2} \text {, }
$$

and the evaluation of paragraph 3 yields

$$
\left|P_{n}^{\prime}(0)\right| \leqq M K(\log n)^{2} .
$$

It is interesting to note that the tangent to the cardioid at the origin is of order less than unity, in the usual sense.

DURE UNIVERSITY 